\title{
Studies on concave Young functions
}

\author{
N. K. Agbeko
}




\title{
STUDIES ON CONCAVE YOUNG-FUNCTIONS
}

\author{
N. K. AGBEKO
}

[Received: November 26, 2004]

\begin{abstract}
Aвstract. We succeeded in isolating a special class of concave Young-functions enjoying the so-called density-level property. In this class there is a proper subset whose members have each the so-called degree of contraction denoted by $c^{*}$, and map bijectively the interval $\left[c^{*}, \infty\right)$ onto itself. We constructed the fixed point of each of these functions. Later we proved that every positive number $b$ is the fixed point of a concave Young-function having $b$ as degree of contraction. We showed that every concave Young-function is square integrable with respect to a specific Lebesgue measure. We also proved that the distance generated by the $L^{2}$-norm is a metric in the set of concave Young-functions and then derived that the concave Young-functions possessing the density-level property constitute a dense set in the space of concave Young-functions.

Mathematics Subject Classification: Primary 47H10, 47H11, 26A18; Secondary 26A06, 26A09, 33B30, 37C25

Keywords: concave Young-functions, degree of contraction, fixed points, dense set
\end{abstract}

\section{INTRODUCTION}

Let $\varphi:(0, \infty) \rightarrow(0, \infty)$ be a right-continuous and decreasing function such that it is integrable on every finite interval $(0, x)$. It is easily seen that the function $\Phi$ : $[0, \infty) \rightarrow[0, \infty)$, defined by

$$
\Phi(x)=\int_{0}^{x} \varphi(t) d t,
$$

is a nonnegative, increasing and concave function with $\Phi(0)=0$. We further assume that $\Phi(\infty)=\infty$ ( $\Phi$ is referred to as concave Young-function in the literature [4].) We note that if $\Phi$ is a concave Young-function, then so is $b \Phi$ for all positive constants $b$. We shall recall the following definition and result in [1].

Definition A. We say that for the concave Young-function $\Phi$ the maximal inequality is valid with some positive constant $K_{\Phi}$ (depending only on $\Phi$ ) if for an arbitrary nonnegative submartingale $\left(X_{n}, \mathcal{F}_{n}\right), n \geq 1$, the inequality

$$
E \Phi\left(X_{n}^{*}\right) \leq K_{\Phi}\left(1+E X_{n}\right)
$$


holds for all $n \geq 1$, where $X_{n}^{*}=\max _{1 \leq k \leq n} X_{k}$.

Theorem B. Let $\Phi$ be any concave Young-function. In order that $\Phi$ satisfy the maximal inequality, it is necessary and sufficient that

$$
A_{\Phi}(\infty):=\int_{1}^{\infty} \frac{\varphi(t)}{t} d t<\infty .
$$

Moreover, if $A_{\Phi}(\infty)<\infty$, then $K_{\Phi}=\max \left(\Phi(1), A_{\Phi}(\infty)\right)$.

Theorem C ([3, p. 205]). Each non-empty subset $B$ of a metric space $X$ is a metric space, the distance in $B$ being the same as in $X$.

We shall say that a concave Young-function $\Phi$ satisfies the density-level property if $A_{\Phi}(\infty)<\infty$. The quantity $A_{\Phi}(\infty)$ will be referred to as density-level and the function $A_{\Phi}:[1, \infty) \rightarrow[0, \infty)$, defined by

$$
A_{\Phi}(x)=\int_{1}^{x} \frac{\varphi(t)}{t} d t
$$

will be called density-level function.

For instance the concave Young-functions $\Phi_{1}(x)=\sqrt{x}$ and $\Phi_{2}(x)=\ln (x+1)$, defined for $x \in[0, \infty)$, have finite density-levels. The concave Young-function $\Phi_{3}(x)=2 x+1-e^{-x}$ is of infinite density-level. In fact, if we let $\varphi_{3}(x)$ stand for the derivative of function $\Phi_{3}(x)$, then

$$
A_{\Phi_{3}}(\infty)=\int_{1}^{\infty} \frac{\varphi_{3}(t)}{t} d t \geq \int_{1}^{\infty} \frac{2}{t} d t=\infty .
$$

Theorem B suggests that the set of concave Young-functions that satisfy the densitylevel property is a rather broad class.

Define function $A_{\Phi}^{*}:(0, \infty) \rightarrow(0, \infty]$ by

$$
A_{\Phi}^{*}(b)=\int_{b}^{\infty} \frac{\varphi(x)}{x} d x,
$$

where $\Phi \in \mathcal{Y}_{\text {conc }}$.

It is not difficult to see that $A_{\Phi_{1}}^{*}(b)<\infty$ and $A_{\Phi_{3}}^{*}(b)=\infty$, for any number $b \in$ $(0, \infty)$, where functions $\Phi_{1}(x)=\sqrt{x}$ and $\Phi_{3}(x)=2 x+1-e^{-x}$ are defined for $x \in[0, \infty)$.

Remark 1. The function $x^{-1} \Phi(x)$ is decreasing on the interval $(0, \infty)$ and

$$
0 \leq \lim _{x \rightarrow \infty} \frac{\Phi(x)}{x}<\infty
$$

Notation. $\mathcal{Y}_{\text {conc }}$ will stand for the collection of all concave Young-functions. $\mathcal{A}$ will denote the set of all functions $\Phi \in \mathcal{Y}_{\text {conc }}$ that satisfy the density-level property.

We note that $\mathcal{A}$ is a proper subset of $\boldsymbol{y}_{\text {conc }}$, since the concave Young-function $\Phi_{3}:[0, \infty) \rightarrow[0, \infty)$, defined above by $\Phi_{3}(x)=2 x+1-e^{-x}$, was shown to be of infinite density-level. 
We recall the following fact: A function $T$ from a metric space $(M, \varrho)$ to itself is called a contraction if there is an $\alpha$ which satisfies $0 \leq \alpha<1$ so that

$$
\varrho(T(x), T(y)) \leq \alpha \varrho(x, y)
$$

for all $x, y \in M$.

We also recall the following well-known principle.

Contraction Mapping Principle ([5]). Let $T$ be a contraction on a complete metric space $(M, \varrho)$. Then there is a unique point $x \in M$ (called fixed point) such that $T(x)=x$. Furthermore, if $x_{0}$ is any point in $M$ and we define $x_{n+1}=T\left(x_{n}\right)$, then $\lim _{n \rightarrow \infty} x_{n}=x$.

In this communication we study, among others, the closure of $\mathcal{A}$ under the composition operation. In a sense, Theorems 1 and 2 show that the concave Young-functions with the density-level property behave like left and right ideals with respect to the composition operation. We also realize that not every function $\Phi \in \mathcal{A}$ admits a fixed point. The investigation in this direction leads us to isolate a proper subset $\mathcal{A}_{1}$ of $\mathcal{A}$ such that every function $\Phi \in \mathcal{A}_{1}$ possesses the so-called degree of contraction, which is closely related to the fixed point of $\Phi$ if it exists. We show that every concave Young-function is square integrable with respect to a specific given Lebesgue measure, and we prove that the natural distance defined by the $L^{2}$-norm satisfies the metric axioms in $\mathcal{Y}_{\text {conc }}$. We then demonstrate that the subset $\mathcal{A}$ proves to be a dense set in $\mathcal{Y}_{\text {conc }}$.

\section{The CLOSURE OF $\mathcal{A}$ UNDER ADDITION AND COMPOSITION OPERATIONS}

Remark 2. For every number $s \in(0, \infty)$ we have that $s \varphi(s)<\Phi(s)$.

Proof. Fix arbitrarily two numbers $s \in(0, \infty)$ and $b \in(0, s)$. Then by applying twice the fact that $\varphi$ decreases on $(0, \infty)$, we have that

$$
\begin{aligned}
\Phi(s) & =\int_{0}^{s} \varphi(t) d t=\int_{0}^{b} \varphi(t) d t+\int_{b}^{s} \varphi(t) d t \geq b \varphi(b)+(s-b) \varphi(s) \\
& >b \varphi(s)+(s-b) \varphi(s)=s \varphi(s),
\end{aligned}
$$

as required.

The following remark is an immediate consequence of Theorem B.

Remark 3. Let $\Phi \in \mathcal{Y}_{\text {conc. If }} \Phi \in \mathcal{A}$, then $\Phi(x) \leq K_{\Phi}(1+x)$ for all $x \in(0, \infty)$, where $K_{\Phi}=\max \left(\Phi(1), A_{\Phi}(\infty)\right)$.

Remark 4. The composition of two concave Young-functions is also a concave Young-function.

The following two lemmas are trivial.

Lemma 1. For any number $b \in(0, \infty)$ and function $\Phi \in \mathcal{Y}_{\text {conc }}$, we have that $b \Phi \in \mathcal{A}$ if and only if $\Phi \in \mathcal{A}$. Moreover, $A_{b \Phi}(x)=b A_{\Phi}(x), x \in[1, \infty)$. 
Lemma 2. Let functions $\Phi_{1}$ and $\Phi_{2} \in \mathcal{Y}_{\text {conc }}$ be arbitrary. Then $\Phi_{1}$ and $\Phi_{2} \in \mathcal{A}$ if and only if $\Phi_{1}+\Phi_{2} \in \mathcal{A}$. Furthermore,

$$
A_{\Phi_{1}+\Phi_{2}}(x)=A_{\Phi_{1}}(x)+A_{\Phi_{2}}(x), \quad x \in[1, \infty) .
$$

Theorem 1. Let functions $\Phi_{1}$ and $\Phi_{2} \in \mathcal{Y}_{\text {conc }}$ be arbitrary. If $\Phi_{2} \in \mathcal{A}$, then $\Phi_{1} \circ \Phi_{2} \in \mathcal{A}$.

Proof. Write $\varphi_{i}$ for the derivative of $\Phi_{i}(i \in\{1,2\})$. Compute the density-level of the composition $\Phi_{1} \circ \Phi_{2}$.

$$
\begin{aligned}
A_{\Phi_{1} \circ \Phi_{2}}(\infty) & =\int_{1}^{\infty} \frac{\varphi_{2}(x) \varphi_{1}\left(\Phi_{2}(x)\right)}{x} d x \\
& \leq \varphi_{1}\left(\Phi_{2}(1)\right) \int_{1}^{\infty} \frac{\varphi_{2}(x)}{x} d x=\varphi_{1}\left(\Phi_{2}(1)\right) A_{\Phi_{2}}(\infty)<\infty,
\end{aligned}
$$

via the monotonicity of function $\varphi_{1}$.

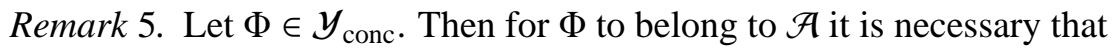

$$
\lim _{t \rightarrow \infty} \varphi(t)=0 .
$$

Proof. Assume that $\Phi \in \mathcal{A}$ but $\lim _{t \rightarrow \infty} \varphi(t)=l_{0}>0$. Pick an arbitrarily fixed number $t \in(1, \infty)$. Then

$$
\infty>A_{\Phi}(\infty) \geq \int_{1}^{t} \frac{\varphi(x)}{x} d x \geq \varphi(t) \log (t)>l_{0} \log (t) .
$$

Passing to the limit, it will follow that $\infty=A_{\Phi}(\infty)<\infty$, which is absurd. This completes the proof.

The following remark suggests that if $\Phi \in \mathcal{Y}_{\text {conc }}$, then either $A_{\Phi}^{*}(b)=\infty$ for all $b \in(0, \infty)$, or $A_{\Phi}^{*}(b)<\infty$ for all $b \in(0, \infty)$.

Remark 6. Let $\Phi \in \mathcal{Y}_{\text {conc }}$. Then $A_{\Phi}^{*}(b)<\infty$ for every constant $b \in(0, \infty) \backslash\{1\}$ if and only if $A_{\Phi}(\infty)<\infty$.

Proof. A simple computation shows that

$$
A_{\Phi}^{*}(b)=\int_{b}^{\infty} \frac{\varphi(x)}{x} d x= \begin{cases}A_{\Phi}(\infty)+\int_{b}^{1} \frac{\varphi(x)}{x} d x & \text { if } b<1 \\ A_{\Phi}(\infty)-\int_{1}^{b} \frac{\varphi(x)}{x} d x & \text { if } b>1,\end{cases}
$$

which yields the result.

Theorem 2. Let functions $\Phi_{1}$ and $\Phi_{2} \in \mathcal{Y}_{\text {conc }}$ be arbitrary. If $\Phi_{1} \in \mathcal{A}$, then $\Phi_{1} \circ \Phi_{2} \in \mathcal{A}$.

Proof. We first show that

$$
A_{\Phi_{1}}(\infty)=\int_{\Phi_{2}^{-1}(1)}^{\infty} \frac{\varphi_{2}(t) \varphi_{1}\left(\Phi_{2}(t)\right)}{\Phi_{2}(t)} d t
$$


where $\Phi_{2}^{-1}$ is the inverse function of $\Phi_{2}$ (whose existence is guaranteed by the continuity of $\Phi_{2}$ ).

In fact, by definition we have that

$$
A_{\Phi_{1}}(\infty)=\int_{1}^{\infty} \frac{\varphi_{1}(x)}{x} d x
$$

Now, setting $x=\Phi_{2}(t)$ we observe that $d x=\varphi_{2}(t) d t$ and thus

$$
A_{\Phi_{1}}(\infty)=\int_{\Phi_{2}^{-1}(1)}^{\infty} \frac{\varphi_{2}(t) \varphi_{1}\left(\Phi_{2}(t)\right)}{\Phi_{2}(t)} d t .
$$

Next, compute the density-level of the composition $\Phi_{1} \circ \Phi_{2}$. Remark 1 implies that

$$
\begin{aligned}
A_{\Phi_{1} \circ \Phi_{2}}(\infty) & =\int_{1}^{\infty} \frac{\varphi_{2}(t) \varphi_{1}\left(\Phi_{2}(t)\right)}{t} d t \\
& =\int_{1}^{\infty} \frac{\Phi_{2}(t)}{t} \frac{\varphi_{2}(t) \varphi_{1}\left(\Phi_{2}(t)\right)}{\Phi_{2}(t)} d t \\
& \leq c \int_{\Phi_{2}^{-1}(1)}^{\infty} \frac{\varphi_{2}(t) \varphi_{1}\left(\Phi_{2}(t)\right)}{\Phi_{2}(t)} d t=c A_{\Phi_{1}}(\infty),
\end{aligned}
$$

where $c=1 / \Phi_{2}^{-1}(1)$ (the second equality holds because of the claim shown above), which was to be proven.

Corollary 1. Let $\Phi \in \mathcal{Y}_{\text {conc }}$ and $\alpha \in(0,1)$ be arbitrary. Then $\Phi_{\alpha} \in \mathcal{A}$, where the function $\Phi_{\alpha}:[0, \infty) \rightarrow[0, \infty)$ is defined by $\Phi_{\alpha}(x)=\Phi^{\alpha}(x)=(\Phi(x))^{\alpha}$.

Proposition 1. Let $\Phi \in \mathcal{Y}_{\text {conc }}$ be arbitrary and fix any number $s \in(0, \infty)$. Then

$$
|\Phi(x)-\Phi(y)| \leq \varphi(s)|x-y|
$$

for all numbers $x, y \in[s, \infty)$.

Proof. Pick numbers $x, y \in[s, \infty)$ arbitrarily. Via the monotonicity of $\Phi$ it follows that

$$
\begin{aligned}
|\Phi(x)-\Phi(y)| & =\max (\Phi(x), \Phi(y))-\min (\Phi(x), \Phi(y)) \\
& =\Phi(\max (x, y))-\Phi(\min (x, y)) .
\end{aligned}
$$

Hence the monotonicity of $\varphi$ yields that

$$
|\Phi(x)-\Phi(y)|=\int_{\min (x, y)}^{\max (x, y)} \varphi(t) d t \leq \varphi(s)(\max (x, y)-\min (x, y))=\varphi(s)|x-y| .
$$

This was to be proved.

We shall similarly show the following proposition.

Proposition 2. Let $\Phi \in \mathcal{A}$ be arbitrary and fix any number $s \in[1, \infty)$. Then

$$
\left|A_{\Phi}(x)-A_{\Phi}(y)\right| \leq \varphi(s)|x-y|
$$

for all numbers $x, y \in[s, \infty)$. 
Proof. Pick numbers $x, y \in[s, \infty)$ arbitrarily. Via the monotonicity of $A_{\Phi}$ it follows that

$$
\begin{aligned}
\left|A_{\Phi}(x)-A_{\Phi}(y)\right| & =\max \left(A_{\Phi}(x), A_{\Phi}(y)\right)-\min \left(A_{\Phi}(x), A_{\Phi}(y)\right) \\
& =A_{\Phi}(\max (x, y))-A_{\Phi}(\min (x, y)) \\
& =\int_{\min (x, y)}^{\max (x, y)} \frac{\varphi(t)}{t} d t \leq \frac{\varphi(s)}{s}|x-y| \leq \varphi(s)|x-y|,
\end{aligned}
$$

because $t^{-1} \varphi(t)$ is a decreasing function.

Proposition 3. Let $x, y \in(0, \infty)$ and $\Delta \subset \mathcal{Y}_{\text {conc }}$ (with $\left.\Delta \neq \varnothing\right)$ be arbitrary. Then

$$
\left|\sup _{\Phi \in \Delta} \Phi(x)-\sup _{\Phi \in \Delta} \Phi(y)\right| \leq \sup _{\Phi \in \Delta}|\Phi(x)-\Phi(y)|,
$$

provided that $\sup _{\Phi \in \Delta} \Phi(t)<\infty$ for all $t \in(0, \infty)$.

Proof. We first note that

$$
\Phi(x) \leq|\Phi(x)-\Phi(y)|+\Phi(y) \text { and } \Phi(y) \leq|\Phi(x)-\Phi(y)|+\Phi(x) .
$$

Taking the supremum we can easily observe that

$$
\sup _{\Phi \in \Delta} \Phi(x) \leq \sup _{\Phi \in \Delta}|\Phi(x)-\Phi(y)|+\sup _{\Phi \in \Delta} \Phi(y)
$$

and

$$
\sup _{\Phi \in \Delta} \Phi(y) \leq \sup _{\Phi \in \Delta}|\Phi(x)-\Phi(y)|+\sup _{\Phi \in \Delta} \Phi(x) .
$$

Combining these inequalities we have that

$$
-\sup _{\Phi \in \Delta}|\Phi(x)-\Phi(y)| \leq \sup _{\Phi \in \Delta} \Phi(x)-\sup _{\Phi \in \Delta} \Phi(y) \leq \sup _{\Phi \in \Delta}|\Phi(x)-\Phi(y)|,
$$

which yields the result.

We know that $k \Phi \in \mathcal{Y}_{\text {conc }}$ for any fixed $\Phi \in \mathcal{Y}_{\text {conc }}$ and all $k \geq 1$. Then

$$
\sup _{\Phi \in \mathcal{Y}_{\text {conc }}} \Phi(x) \geq \sup _{k \geq 1} k \Phi(x)= \begin{cases}0 & \text { if } x=0, \\ \infty & \text { if } x \in(0, \infty),\end{cases}
$$

meaning that there is no real function $g(x)$ such that $\Phi(x) \leq g(x)$ for all $\Phi \in \mathcal{Y}_{\text {conc }}$ and $x \in[0, \infty)$. Nevertheless, this is possible for their suitably normalised forms, as shown in the following lemma.

Lemma 3. The function $S:[0, \infty) \rightarrow[0, \infty)$, defined by

$$
S(x)=\sup _{\Phi \in \mathcal{Y}_{\text {conc }}}(\Phi(1))^{-1} \Phi(x),
$$

has the following properties:

(1) $S(0)=0$ and $S(1)=1$. 
(2) $S$ is a non-decreasing function such that

$$
(\Phi(1))^{-1} \Phi(x) \leq S(x)
$$

for all $\Phi \in \mathcal{Y}_{\text {conc }}$ and $x \in[0, \infty)$.

(3) The identity

$$
\sup _{\Phi \in \mathcal{Y}_{\text {conc }}}(1+\Phi(1))^{-1}=1
$$

holds.

(4) For every number $x \in[0, \infty)$, the chain of inequalities $x \leq S(x) \leq x+1$ holds true.

(5) We have that $\lim _{x \rightarrow \infty} x^{-1} S(x)=1$ and $\lim _{x \rightarrow \infty} S(x)=\infty$.

Proof. The first part is obvious. We show that $S(x)$ is a non-decreasing function. In fact, pick arbitrarily two numbers $x_{1}$ and $x_{2} \in[0, \infty)$ with $x_{1}<x_{2}$. By the monotonicity we have that $\Phi\left(x_{1}\right)<\Phi\left(x_{2}\right)$. If we normalize this inequality suitably and then take the supremum on both sides over all $\Phi \in \mathcal{Y}_{\text {conc }}$ we can then observe that $S\left(x_{1}\right) \leq S\left(x_{2}\right)$. Thus $S$ is a non-decreasing function. To show the identity in the third part we begin by establishing the inequality $(1+\Phi(1))^{-1} \leq 1$, which holds for every $\Phi \in \mathcal{Y}_{\text {conc }}$. Then $\sup _{\Phi \in \mathcal{Y}_{\text {conc }}}(1+\Phi(1))^{-1} \leq 1$. We also know that $k^{-1} \Phi \in \mathcal{Y}_{\text {conc }}$ for any fixed integer $k \geq 1$. Hence

$$
\left(1+k^{-1} \Phi(1)\right)^{-1} \leq \sup _{\Phi \in \mathcal{Y}_{\text {conc }}}(1+\Phi(1))^{-1} .
$$

Passing to the limit we observe that $\lim _{k \rightarrow \infty}\left(1+k^{-1} \Phi(1)\right)^{-1}=1$. Consequently $\sup _{\Phi \in \mathcal{Y}_{\text {conc }}}(1+\Phi(1))^{-1}=1$. The fourth part will be proved if we show that $S(x) \leq$ $x+1$ and $S(x) \geq x$ for all $x \in[0, \infty)$. In fact, take arbitrarily a function $\Phi \in \mathcal{y}_{\text {conc. }}$. Clearly the equation of the tangent line of $\Phi$ at the point $(1, \Phi(1))$ is given by $y=\varphi(1)(x-1)+\Phi(1), x \in[0, \infty)$. Via the concavity of $\Phi$, it is obvious that $\Phi(x) \leq \varphi(1)(x-1)+\Phi(1), x \in[0, \infty)$. Hence by Remark 2 we have $\Phi(x) \leq$ $\varphi(1) x+\Phi(1)<\Phi(1)(x+1), x \in[0, \infty)$. This implies that $S(x)<x+1$, for all $x \in[0, \infty)$. Finally fix arbitrarily a function $\Phi \in \mathcal{Y}_{\text {conc. Then the function, defined on }}$ $[0, \infty)$ by $x+\Phi(x)$ (for any fixed $\Phi \in \mathcal{Y}_{\text {conc }}$ ), also belongs to $\mathcal{Y}_{\text {conc }}$. Hence

$$
S(x) \geq \frac{x+\Phi(x)}{1+\Phi(1)} \geq \frac{x}{1+\Phi(1)}, x \in[0, \infty) .
$$

Now taking the supremum over $\Phi \in \mathcal{Y}_{\text {conc }}$, the third part leads to the desired inequality $S(x) \geq x$. To complete the proof we just point out that the fifth part becomes obvious because of the fourth part.

Lemma 4. The function $H:[1, \infty) \rightarrow[0, \infty)$ defined by

$$
H(x)=\sup _{\Phi \in \mathcal{A}}(\varphi(1))^{-1} \Phi(x)
$$


is increasing and has the property that

$$
|H(x)-H(y)| \leq|x-y|
$$

for all $x, y \in[1, \infty)$.

\section{The fixed points of a Class of concave Young-functions}

We shall introduce the following notion (probably new, at least in the author's opinion). Some series of examples will justify that it is well founded.

Definition 1. Let $\Phi \in \mathcal{A}$ be arbitrary. The number $c^{*} \in(0, \infty)$ will be called the degree of contraction of function $\Phi$ if there is some constant $\alpha>1$ such that both the identities

$$
\int_{c^{*}}^{\infty} \frac{\varphi(t)}{t} d t=1 \text { and } \int_{c^{*}}^{\alpha c^{*}} \frac{\varphi(t)}{t} d t=\varphi\left(c^{*}\right)
$$

hold simultaneously. (In this case we shall say that $\Phi$ admits the number $c^{*}$ as its degree of contraction.)

Example 1. For $\Phi(x)=\sqrt{x+1}-1, x \in[0, \infty)$, the degree of contraction of $\Phi$ is equal to $\frac{4 e^{2}}{e^{4}-2 e^{2}+1} \approx 0.7240616609$ with

$$
\alpha=\frac{\left(e^{2}-1\right)^{2} e^{2 /\left(e^{2}+1\right)-1}}{e^{4 /\left(e^{2}+1\right)+2}-2 e^{2 /\left(e^{2}+1\right)+1}+1} \approx 3.175019732 .
$$

Example 2. For any fixed number $p \in(0,1)$, the degree of contraction of the function $\Phi_{p}(x)=x^{p}, x \in[0, \infty)$, is equal to $\left(\frac{p}{1-p}\right)^{\frac{1}{1-p}}$ with $\alpha=\frac{1}{p^{1 /(1-p)}}$.

Example 3. The degree of contraction of the function $\log (x+1), x \in[0, \infty)$, equals $(e-1)^{-1}$ with $\alpha=\frac{e-1}{e^{1 / e}-1} \approx 3.864191634$.

Proposition 4. Let $\Phi \in \mathcal{A}$ admit the number $c^{*}$ as its degree of contraction. Then $\varphi\left(c^{*}\right)<1$.

Proof. By Definition 1, there is a number $\alpha>1$ such that both the identities

$$
\int_{c^{*}}^{\infty} \frac{\varphi(t)}{t} d t=1 \text { and } \int_{c^{*}}^{\alpha c^{*}} \frac{\varphi(t)}{t} d t=\varphi\left(c^{*}\right)
$$

hold simultaneously. Consequently we have that

$$
1=\int_{c^{*}}^{\infty} \frac{\varphi(t)}{t} d t=\int_{c^{*}}^{\alpha c^{*}} \frac{\varphi(t)}{t} d t+\int_{\alpha c^{*}}^{\infty} \frac{\varphi(t)}{t} d t>\int_{c^{*}}^{\alpha c^{*}} \frac{\varphi(t)}{t} d t=\varphi\left(c^{*}\right)
$$

because

$$
0<\int_{\alpha c^{*}}^{\infty} \frac{\varphi(t)}{t} d t<\infty
$$

by the assumption and the monotonicity of the function $t^{-1} \varphi(t)$ on the interval $(0, \infty)$. This was to be proved. 
On the one hand it is not difficult to verify that 1 is the degree of contraction of function $\Phi(x)=\sqrt{x}, x \in[0, \infty)$, with $\alpha=4$, and 1 is the unique solution of equation $\Phi(x)=x$ on interval $[1, \infty)$. On the other hand, we know, for instance, in Example 3, that $(e-1)^{-1}$ is the degree of contraction of function $\log (x+1)$. Nevertheless,

$$
\log \left(\frac{1}{e-1}+1\right)=\log \left(\frac{e}{e-1}\right) \neq \frac{1}{e-1} .
$$

The question thus arises which are those functions $\Phi \in \mathcal{A}$ that are contractions. We shall provide a proper subset of $\mathcal{A}$ enjoying this property.

Theorem 3. Let $\Phi \in \mathcal{Y}_{\text {conc }}$ and $c^{*}$ be any positive number. In order that the equality $\Phi\left(c^{*}\right)=c^{*}$ hold, it is necessary and sufficient that the range of the function $\left.\Phi\right|_{\left[c^{*}, \infty\right)}:\left[c^{*}, \infty\right) \rightarrow[0, \infty)$, defined by the fotmula

$$
\left.\Phi\right|_{\left[c^{*}, \infty\right)}(x)=\Phi(x),
$$

should equal the interval $\left[c^{*}, \infty\right)$.

Proof. Suppose that $\left.\Phi\right|_{\left[c^{*}, \infty\right)}\left(c^{*}\right)=\Phi\left(c^{*}\right)=c^{*}$. Obviously $\Phi$ is a bijection on $[0, \infty)$. Hence it follows that $\left.\Phi\right|_{\left[c^{*}, \infty\right)}$ is an injection on $\left[c^{*}, \infty\right)$. Since $\left.\Phi\right|_{\left[c^{*}, \infty\right)}$ is continuous on $\left[c^{*}, \infty\right)$ and tends increasingly to $\infty$, we have that the range of function $\left.\Phi\right|_{\left[c^{*}, \infty\right)}$ equals $\left[\Phi\left(c^{*}\right), \infty\right)=\left[c^{*}, \infty\right)$, by assumption. Conversely, assume that the range of $\left.\Phi\right|_{\left[c^{*}, \infty\right)}$ equals interval $\left[c^{*}, \infty\right)$, but, on the contrary, there is some number $y \in\left(c^{*}, \infty\right)$ such that $\Phi(y)=\left.\Phi\right|_{\left[c^{*}, \infty\right)}(y)=c^{*}$. By the assumption it is obvious that function $\left.\Phi\right|_{\left[c^{*}, \infty\right)}$ is surjective on $\left[c^{*}, \infty\right)$. Moreover, $\left.\Phi\right|_{\left[c^{*}, \infty\right)}$ maps bijectively the interval $\left[c^{*}, \infty\right)$ onto itself because it is also an injection. The monotonicity of $\Phi$ yields that $\Phi\left(c^{*}\right)=\left.\Phi\right|_{\left[c^{*}, \infty\right)}\left(c^{*}\right)<\Phi(y)=c^{*}$. However, by the bijective property of $\left.\Phi\right|_{\left[c^{*}, \infty\right)}$, we have that $\Phi\left(c^{*}\right) \geq c^{*}$. Consequently the inequality $c^{*}<c^{*}$ follows. This, however, is absurd. Therefore, we can conclude on the validity of the argument.

Notation. $\mathcal{A}_{1}$ will stand for the collection of all functions $\Phi \in \mathcal{A}$ mapping bijectively the interval $\left[c^{*}, \infty\right)$ onto itself, where $c^{*}$ is the degree of contraction of $\Phi$.

Theorem 4. Let $\Phi \in \mathcal{A}_{1}$ with $c^{*}$ its degree of contraction. Endow the interval $\left[c^{*}, \infty\right)$ with the metric $\varrho(\cdot, \cdot):\left[c^{*}, \infty\right) \times\left[c^{*}, \infty\right) \rightarrow[0, \infty)$ defined by $\varrho(x, y)=|x-y|$. Then $\Phi$ is a contraction over the metric space $\left(\left[c^{*}, \infty\right), \varrho\right)$.

Moreover, the number $c^{*}$ is the unique solution of the equation $\Phi(x)=x$ on $\left[c^{*}, \infty\right)$.

Proof. We first note that the pair $\left(\left[c^{*}, \infty\right), \varrho\right)$ is a complete metric space. Combining Propositions 1 and 4 we can easily derive that $\Phi$ is a contraction on the metric space $\left(\left[c^{*}, \infty\right), \varrho\right.$ ). But since $\Phi\left(c^{*}\right)=c^{*}$ (via Theorem 3), we deduce, referring to the Contraction Mapping Principle, that the degree of contraction $c^{*}$ is the unique solution to the equation $\Phi(x)=x$ on the interval $\left[c^{*}, \infty\right)$. This completes the proof. 
Theorem 5. For every number $b \in(0, \infty)$ there can be found some function $\Phi_{b} \in$ $\mathcal{A}_{1}$ with degree of contraction $b$. Furthermore, number $b$ is the unique solution of equation $\Phi_{b}(x)=x$ on the interval $[b, \infty)$.

Proof. Let $b \in(0, \infty)$ be any number and define the function $\Phi_{b}(t)=\sqrt{b t}, t \in$ $[0, \infty)$. Clearly, the derivative of $\Phi_{b}(t)$ is expressed by $\varphi(t)=\frac{\sqrt{b}}{2 \sqrt{t}}, t \in(0, \infty)$. On the one hand, an easy calculation shows that

$$
\int_{b}^{\infty} \frac{\varphi(t)}{t} d t=\sqrt{b} \int_{b}^{\infty} \frac{1}{2 t \sqrt{t}} d t=\sqrt{b} \lim _{x \rightarrow \infty}\left(\frac{1}{\sqrt{b}}-\frac{1}{\sqrt{x}}\right)=1
$$

and

$$
\int_{b}^{4 b} \frac{\varphi(t)}{t} d t=\sqrt{b} \int_{b}^{4 b} \frac{1}{2 t \sqrt{t}} d t=\frac{1}{2}=\varphi(b)
$$

i. e., number $b$ is the degree of contraction of $\Phi_{b}$. On the other hand, an easy substitution leads to

$$
\Phi_{b}(b)=\sqrt{b^{2}}=b .
$$

Then Theorem 3 implies that $\Phi_{b} \in \mathcal{A}_{1}$. Consequently Theorem 4 entails that $\Phi_{b}$ is a contraction over the metric space $([b, \infty), \varrho)$ and moreover, number $b$ is the unique solution of equation $\Phi_{b}(x)=x$ on $[b, \infty)$, with $\varrho$ being the metric induced by the absolute value function. This concludes the proof.

To end this section we should like to point out that the set

$$
\left\{\Phi \in \mathcal{Y}_{\text {conc }} \backslash \mathcal{A}: \Phi \text { admits a positive fix point }\right\}
$$

is a non-empty set. In fact, it is not hard to check that the function $\Phi$, defined by $\Phi(x)=\frac{x}{2}+\sqrt{x}$ whenever $x \in[0, \infty)$, belongs to $\mathcal{Y}_{\text {conc }} \backslash \mathcal{A}$ and $\Phi(4)=4$.

\section{IS THE SET $\mathcal{A}$ DENSE IN $\boldsymbol{Y}_{\text {conc }}$ ?}

We shall answer this question in the affirmative.

Theorem 6. For any concave Young-function $\Phi$, there exists a sequence $\left(\Phi_{n}\right) \subset \mathcal{A}$ such that $\left(\Phi_{n}\right)$ converges pointwise to $\Phi$, i. e., $\lim _{n \rightarrow \infty} \Phi_{n}(x)=\Phi(x)$ whenever $x \in$ $[0, \infty)$.

Proof. Fix arbitrarily an index $n \geq 1$ and define $\Phi_{n}(x)=\Phi^{n /(n+1)}(x), x \in[0, \infty)$. Obviously, $\left(\Phi_{n}\right) \subset \mathcal{Y}_{\text {conc }}$ because of Remark 4. So, on the one hand, Corollary 1 yields that $\left(\Phi_{n}\right) \subset \mathcal{A}$. On the other hand, we can easily see in the limit that

$$
\lim _{n \rightarrow \infty} \Phi_{n}(x)=\lim _{n \rightarrow \infty} \Phi^{n /(n+1)}(x)=\Phi(x)
$$

for every $x \in[0, \infty)$. Therefore, we conclude on the validity of the theorem.

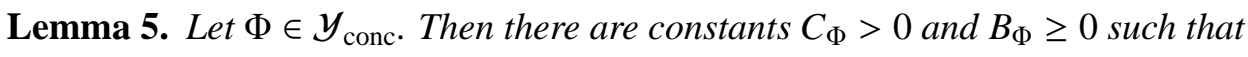

$$
A_{\Phi}(\infty)-B_{\Phi} \leq \int_{0}^{\infty} \frac{\Phi(t)}{(t+1)^{2}} d t \leq C_{\Phi}+A_{\Phi}(\infty)
$$


Proof. An integration by parts leads to

$$
\int_{0}^{\infty} \frac{\Phi(t)}{(t+1)^{2}} d t=\left[\frac{-\Phi(t)}{t+1}\right]_{0}^{\infty}+\int_{0}^{\infty} \frac{\varphi(t)}{t+1} d t=\int_{0}^{\infty} \frac{\varphi(t)}{t+1} d t-B_{\Phi},
$$

where $0 \leq B_{\Phi}:=\lim _{t \rightarrow \infty} \frac{\Phi(t)}{t+1}<\infty$, as $\frac{\Phi(t)}{t+1}<\frac{\Phi(t)}{t}$ for all $t \in(0, \infty)$. On the one hand,

$$
\int_{0}^{\infty} \frac{\varphi(t)}{t+1} d t=\int_{0}^{1} \frac{\varphi(t)}{t+1} d t+\int_{1}^{\infty} \frac{\varphi(t)}{t+1} d t \leq \int_{0}^{1} \frac{\varphi(t)}{t+1} d t+A_{\Phi}(\infty)
$$

On the other hand, by the monotonicity of function $\varphi(t)$ and by the change of variables, we have that

$$
\int_{0}^{\infty} \frac{\varphi(t)}{t+1} d t \geq \int_{0}^{\infty} \frac{\varphi(t+1)}{t+1} d t=\int_{1}^{\infty} \frac{\varphi(x)}{x} d x=A_{\Phi}(\infty) .
$$

Consequently if we combine (4.1)-(4.3), one can observe that

$$
A_{\Phi}(\infty)-B_{\Phi} \leq \int_{0}^{\infty} \frac{\Phi(t)}{(t+1)^{2}} d t \leq \int_{0}^{1} \frac{\varphi(t)}{t+1} d t+B_{\Phi}+A_{\Phi}(\infty) .
$$

This leads to the desired result.

Lemma 5 suggests that the quantity $\int_{0}^{\infty} \frac{\Phi(t)}{(t+1)^{2}} d t$ and the density-level $A_{\Phi}(\infty)$ are equivalent, in the sense that they are both either finite or infinite. This gives rise to the following essential result.

Lemma 6. Let $\Phi \in \mathcal{Y}_{\text {conc }}$ be arbitrary. Then

$$
\int_{0}^{\infty} \frac{(\Phi(x))^{2}}{(x+1)^{4}} d x<\infty
$$

Proof. Clearly,

$$
\begin{aligned}
\int_{0}^{\infty} \frac{(\Phi(x))^{2}}{(x+1)^{4}} d x & =\int_{0}^{1} \frac{(\Phi(x))^{2}}{(x+1)^{4}} d x+\int_{1}^{\infty} \frac{(\Phi(x))^{2}}{(x+1)^{4}} d x \\
& \leq \int_{0}^{1} \frac{(\Phi(x))^{2}}{(x+1)^{4}} d x+\int_{1}^{\infty} \frac{(\Phi(x))^{2}}{x^{4}} d x
\end{aligned}
$$

Integration by parts yields

$$
\int_{1}^{\infty} \frac{(\Phi(x))^{2}}{x^{4}} d x=\frac{\Phi(1)}{3}+\frac{2}{3} \int_{1}^{\infty} \frac{\varphi(x) \Phi(x)}{x^{3}} d x \leq \frac{\Phi(1)}{3}+\frac{2 \varphi(1) \Phi(1)}{3},
$$

because $\varphi(x)$ and $\frac{\Phi(x)}{x}$ are decreasing functions.

Now endow the half line $[0, \infty)$ with a $\sigma$-algebra $\mathcal{M}$ containing the Borel sets. Define a Lebesgue measure $\mu: \mathcal{M} \rightarrow[0, \infty)$ by setting

$$
\mu([0, x))=\frac{1}{3}\left(1-\frac{1}{(x+1)^{3}}\right)
$$


for all $x \in[0, \infty)$. Let $L^{2}:=L^{2}([0, \infty), \mathcal{M}, \mu)$ be the collection of all (measurable) square integrable functions. We know (see [6, p. 326], Remark 11.37) that the pair $\left(L^{2}, d\right)$ is not a metric space unless we identify functions which differ only on a set of measure zero, where the mapping $d: L^{2} \times L^{2} \rightarrow[0, \infty)$ is defined by

$$
d(f, g)=\sqrt{\int_{[0, \infty)}(f-g)^{2} d \mu}=\sqrt{\int_{0}^{\infty} \frac{(f(x)-g(x))^{2}}{(x+1)^{4}} d x} .
$$

By Lemma 6, we observe that $\mathcal{Y}_{\text {conc }} \subset L^{2}$. Unfortunately, we note that this does not guarantee that the pair $\left(\mathcal{Y}_{\text {conc }}, d\right)$ is a metric space, for the reason mentioned above. Nevertheless, we shall prepare the ground for showing that $\left(\mathcal{Y}_{\text {conc }}, d\right)$ is actually a metric space.

Whenever $\Phi \in \mathcal{Y}_{\text {conc }}$ write $G_{\Phi}$ for the graph of $\Phi$ on $[0, \infty)$, i. e.,

$$
G_{\Phi}=\{(x, \Phi(x)): x \in[0, \infty)\}
$$

and write $G_{\Phi}^{a \| b b}$ for the graph of $\Phi$ on the interval $[a, b)$, i. e.,

$$
G_{\Phi}^{a \| b}=\{(x, \Phi(x)): x \in(a, b)\},
$$

where $a<b$ are any non-negative numbers.

Lemma 7. Let $\Phi$ and $\Psi \in \mathcal{Y}_{\text {conc }}$ be arbitrary with distinct graphs. Then

$$
|\{x \in(0, \infty): \Phi(x)=\Psi(x)\}| \leq 1,
$$

where $|B|$ stands for the cardinality of $B$ whenever $B$ is a set.

Proof. Suppose on the contrary that

$$
|\{x \in(0, \infty): \Phi(x)=\Psi(x)\}| \geq 2 .
$$

Write

$$
x_{1}=\inf \{x \in(0, \infty): \Phi(x)=\Psi(x)\}
$$

and

$$
x_{2}=\inf \left\{x \in(0, \infty) \backslash\left\{x_{1}\right\}: \Phi(x)=\Psi(x)\right\} .
$$

It is clear that $0<x_{1}<x_{2}$ and $\Phi\left(x_{i}\right)=\Psi\left(x_{i}\right), i \in\{1,2\}$. We point out that the two graphs are continuous. We show that the graph of one of the functions $\Phi$ and $\Psi$ lies above the graph of the other on the interval $\left(0, x_{1}\right)$. In fact, without loss of generality we may assume on the contrary that $G_{\Phi}^{0 \| x_{1}}$ lies both above and below $G_{\Psi}^{0 \| x_{1}}$. Then necessarily the two graphs must cross each other in the interior of interval $\left(0, x_{1}\right)$, i. e. there is some $x_{0} \in\left(0, x_{1}\right)$ such that $\Phi\left(x_{0}\right)=\Psi\left(x_{0}\right)$. This, however, is in contradiction with the minimality of $x_{1}$. Hence we can assume that $G_{\Phi}^{0 \| x_{1}}$ lies above $G_{\Psi}^{0 \| x_{1}}$. By the continuity and the fact that $\Phi\left(x_{1}\right)=\Psi\left(x_{1}\right)$ we note that $G_{\Phi}$ crosses $G_{\Psi}$ at point $\left(x_{1}, \Phi\left(x_{1}\right)\right)$. Nevertheless, since both $\Phi$ and $\Psi$ are unbounded increasing functions and $\Phi\left(x_{2}\right)=\Psi\left(x_{2}\right)$, the graph $G_{\Phi}$ must cross the graph $G_{\Psi}$ at 
point $\left(x_{2}, \Phi\left(x_{2}\right)\right)$. This means that $\Phi$ must be convex on the interval $\left(x_{1}, x_{2}\right)$, which is absurd since these functions are concave.

Corollary 2. Let $\Phi$ and $\Psi \in \mathcal{Y}_{\text {conc }}$ be arbitrary. Then among the following three assertions exactly one fulfills

(1) $\{x \in[0, \infty): \Phi(x)=\Psi(x)\}=[0, \infty)$.

(2) $\{x \in(0, \infty): \Phi(x) \neq \Psi(x)\}=(0, \infty)$.

(3) There is a unique number $x^{*} \in(0, \infty)$ with $\Phi\left(x^{*}\right)=\Psi\left(x^{*}\right)$ such that

$$
\left\{x \in(0, \infty) \backslash\left\{x^{*}\right\}: \Phi(x) \neq \Psi(x)\right\}=(0, \infty) \backslash\left\{x^{*}\right\} .
$$

Lemma 8. Let $\Phi$ and $\Psi \in \mathcal{Y}_{\text {conc }}$ be arbitrary. Then in order that $\Phi(x)=\Psi(x)$ for all $x \in[0, \infty)$ it is necessary and sufficient that

$$
\int_{[0, \infty)}(\Phi-\Psi)^{2} d \mu=0
$$

Proof. We first note that the sufficiency is obvious. To show the necessity let us assume that

Then, on the one hand,

$$
\int_{[0, \infty)}(\Phi-\Psi)^{2} d \mu=0
$$

$$
\mu(\{x \in[0, \infty): \Phi(x)=\Psi(x)\})=\mu([0, \infty))=\frac{1}{3}
$$

so that necessarily $\{x \in[0, \infty): \Phi(x)=\Psi(x)\} \neq \varnothing$. On the other hand

$$
\mu(\{x \in(0, \infty): \Phi(x) \neq \Psi(x)\})=0 .
$$

Note that both the sets $\{x \in[0, \infty): \Phi(x)=\Psi(x)\}$ and $\{x \in(0, \infty): \Phi(x) \neq \Psi(x)\}$ cannot be non-empty at the same time (because of Corollary 2). Consequently,

$$
\{x \in(0, \infty): \Phi(x) \neq \Psi(x)\}=\varnothing
$$

and, therefore, $\{x \in[0, \infty): \Phi(x)=\Psi(x)\}=[0, \infty)$.

We are now in the position to state the result hereby.

Proposition 5. The mapping $d: \mathcal{Y}_{\text {conc }} \times \mathcal{Y}_{\text {conc }} \rightarrow[0, \infty)$, defined by

$$
d(\Phi, \Psi)=\sqrt{\int_{[0, \infty)}(\Phi-\Psi)^{2} d \mu}=\sqrt{\int_{0}^{\infty} \frac{(\Phi(x)-\Psi(x))^{2}}{(x+1)^{4}} d x},
$$

satisfies the metric axioms, i. e. for any three functions $\Phi_{1}, \Phi_{2}$ and $\Phi_{3} \in \mathcal{Y}_{\text {conc }}$

(1) $d\left(\Phi_{1}, \Phi_{2}\right) \geq 0$ and $d\left(\Phi_{1}, \Phi_{2}\right)=0$ if and only if $\Phi_{1}=\Phi_{2}$.

(2) $d\left(\Phi_{1}, \Phi_{2}\right)=d\left(\Phi_{2}, \Phi_{1}\right)$.

(3) $d\left(\Phi_{1}, \Phi_{2}\right) \leq d\left(\Phi_{1}, \Phi_{3}\right)+d\left(\Phi_{3}, \Phi_{2}\right)$.

The pair $\left(\boldsymbol{y}_{\text {conc }}, d\right)$ is a metric space and then by referring to Theorem $\mathrm{C}$ the pair $(\mathcal{A}, d)$ is also a metric space. 
Theorem 7. Let $\Phi \in \mathcal{Y}_{\text {conc }}$ and write $\Phi_{n}=\Phi^{n /(n+1)}, n \geq 1$. Then

$$
\lim _{n \rightarrow \infty} \int_{[0, \infty)} \Phi_{n}^{2} d \mu=\int_{[0, \infty)} \Phi^{2} d \mu .
$$

Proof. For every index $n \geq 1$, define $\Phi_{n}^{*}:=\left(\Phi_{n}(1)\right)^{-1} \Phi_{n}$. Clearly $\left(\Phi_{n}\right) \subset \mathcal{Y}_{\text {conc }}$ (see Corollary 1) and hence $\left(\Phi_{n}^{*}\right) \subset \mathcal{A}$ because of Lemma 1. Via Theorem 6 it follows that sequence $\left(\Phi_{n}\right)$ converges to $\Phi$ pointwise, which in turn entails that sequence $\left(\Phi_{n}^{*}\right)$ converges to $(\Phi(1))^{-1} \Phi$ pointwise. Write the function $Z(x):=x+1, x \in[0, \infty)$. We obtain (by Lemma 3 ) that

$$
\sup _{n \geq 1} \Phi_{n}^{*}(x) \leq S(x) \leq Z(x), \quad x \in[0, \infty) .
$$

Now, on the one hand, a simple computation shows that $Z \in L^{2}$. On the other hand, we can deduce from Lemma 6 that $\left(\Phi_{n}\right) \subset L^{2}$ and thus $\left(\Phi_{n}^{*}\right) \subset L^{2}$. Therefore, the Dominated Convergence Theorem guarantees that

$$
\lim _{n \rightarrow \infty} \int_{[0, \infty)} \Phi_{n}^{* 2} d \mu=(\Phi(1))^{-2} \int_{[0, \infty)} \Phi^{2} d \mu .
$$

Now we remark that for every index $n \geq 1$,

$$
\int_{[0, \infty)} \Phi_{n}^{2} d \mu=(\Phi(1))^{2} \int_{[0, \infty)} \Phi_{n}^{* 2} d \mu .
$$

Passing to the limit we can conclude that

$$
\lim _{n \rightarrow \infty} \int_{[0, \infty)} \Phi_{n}^{2} d \mu=\int_{[0, \infty)} \Phi^{2} d \mu .
$$

This was to be proven.

Theorem 8. The subset $\mathcal{A}$ is a dense set in $\mathcal{Y}_{\text {conc. }}$

Proof. Let $\Phi \in \mathcal{Y}_{\text {conc }}$ be arbitrary. For every index $n \geq 1$, set $\Phi_{n}^{*}:=\left(\Phi_{n}(1)\right)^{-1} \Phi_{n}$, where $\Phi_{n}=\Phi^{n /(n+1)}$. We need to prove that

$$
\lim _{n \rightarrow \infty} d\left(\Phi, \Phi_{n}\right)=\lim _{n \rightarrow \infty} \int_{[0, \infty)}\left(\Phi-\Phi_{n}\right)^{2} d \mu=0 .
$$

In fact, fix arbitrarily an index $n \geq 1$. Then

$$
\int_{[0, \infty)}\left(\Phi-\Phi_{n}\right)^{2} d \mu=\int_{[0, \infty)} \Phi_{n}^{2} d \mu+\int_{[0, \infty)} \Phi^{2} d \mu-2 \int_{[0, \infty)} \Phi \Phi_{n} d \mu .
$$

Then Lemma 3 entails that

$$
(\Phi(1))^{-(2 n+1) /(n+1)} \Phi \Phi_{n} \leq Z^{(2 n+1) /(n+1)} \leq Z^{2},
$$

since $Z(x) \geq 1$ for all $x \in[0, \infty)$ and the sequence $\left(\frac{2 n+1}{n+1}\right)$ tends increasingly to 2 . On the other hand,

$$
\lim _{n \rightarrow \infty}(\Phi(1))^{-(2 n+1) /(n+1)} \Phi(x) \Phi_{n}(x)=(\Phi(1))^{-2} \Phi^{2}(x)
$$


for all $x \in[0, \infty)$. Then by means of The Dominated Convergence Theorem it follows that

$$
\begin{aligned}
\lim _{n \rightarrow \infty} \int_{[0, \infty)} \Phi \Phi_{n} d \mu & =\lim _{n \rightarrow \infty}(\Phi(1))^{(2 n+1) /(n+1)} \int_{[0, \infty)}(\Phi(1))^{-\frac{2 n+1}{n+1}} \Phi^{\frac{2 n+1}{n+1}} d \mu \\
& =\int_{[0, \infty)} \Phi^{2} d \mu .
\end{aligned}
$$

Finally we note that

$$
\lim _{n \rightarrow \infty} \int_{[0, \infty)} \Phi_{n}^{2} d \mu=\int_{[0, \infty)} \Phi^{2} d \mu,
$$

by Theorem 7. Therefore, combining the results established in (4.4)-(4.6), we get $\lim _{n \rightarrow \infty} d\left(\Phi, \Phi_{n}\right)=0$. We can thus conclude on the validity of the theorem.

If the integral representation (1.1) is such that its derivative is a right-continuous function, tending increasingly to infinity and assumes the value zero at the origin, then we speak of convex Young-functions (see, e. g. [2]). Clearly the inverse of every convex Young-function is a concave Young-function (and vice versa).

A convex Young-function $\Psi$ is said to satisfy the growth condition if

$$
\sup _{x>0} \frac{\Psi(\beta x)}{\Psi(x)}<\infty
$$

for some number $\beta>1$ which is equivalent to

$$
\sup _{x>0} \frac{x \psi(x)}{\Psi(x)}:=p<\infty
$$

with $\psi$ being the derivative of $\Psi$. (The quantity $p$ is referred to as the power of $\Psi$.)

Open problem 1. Let $\Phi \in \mathcal{Y}_{\text {conc }}$ be arbitrary. In order that $\Phi(x)=x^{p}, x \in[0, \infty)$ for some $p \in(0,1)$ it is necessary and sufficient that both $\Phi \in \mathcal{A}_{1}$ and its inverse $\Phi^{-1}$ satisfy the growth condition together with the property $\Phi^{-1}(1)=1$.

Open problem 2. The converse of Remark 5 holds true.

\section{ACKNOWLEDGEMENT}

The author is thankful to the referee for the suggestions and constructive criticism made about the first draft of this paper, as they were very much edifying. The author also expresses his thanks to Dr. Ferenc Nagy for his valuable comments.

\section{REFERENCES}

[1] Agbeko, N. K.: Necessary and sufficient condition for the maximal inequality of concave Youngfunctions, Annales Univ. Sci. Budapest, Sectio Mathematica, 29 (1989), 267-270.

[2] Krasnosel'skiI, M. A. and Rutitckit, Ya. B.: Convex functions and Orlicz spaces, Noordhoff, Groningen, 1961.

[3] Kuratowski, K.: Topology, Vol. 1, Academic Press, New York, etc., 1966. 
[4] Mogyoródi, J.: On concave function inequality for martingales, Annales Univ. Sci. Sect. Math., 24 (1981), 255-271.

[5] Reed, M. C.: Fundamental ideas of analysis, John Wiley \& Sons, New York, etc., 1998.

[6] Rudin, W.: Principles of mathematical analysis, 3rd ed., McGraw-Hill Book Company, Auckland, Tokyo, etc., 1976.

\section{Author's Address}

\section{N. K. Agbeko:}

Institute of Mathematics, University of Miskolc, H-3515 Miskolc-Egyetemváros, Hungary

E-mail address: matagbek@uni.miskolc.hu 